\title{
On the seismic cycle seen as a relaxation oscillation
}

\author{
T. PUTELAT, ${ }^{\dagger \ddagger *}$ J.R. WILLIS ${ }^{\dagger} \&$ J.H.P. DAWES ${ }^{\dagger}$ \\ $\dagger$ ITG \& DAMTP, Centre for Mathematical Sciences \\ University of Cambridge, Cambridge, UK \\ ¥ LMM, Polytechnic School \\ Aristotle University of Thessaloniki, Thessaloniki, Greece.
}

(Received 02/03/2008)

\begin{abstract}
An earthquake is commonly described as a stick-slip frictional instability occurring along preexisting crustal faults. The seismic cycle of earthquake recurrence is characterised by long periods of quasi-static evolution which precede sudden slip events accompanied by elastic wave radiation: the earthquake. This succession of processes over two well distinguished time scales recalls the behaviour of nonlinear relaxation oscillations. We explore this connection by studying, in the framework of rate-and-state friction, the sliding of two identical slabs of elastic solid driven in opposite directions with a constant relative velocity. Our first innovation is to establish that the motion of a spring-block system is an asymptotic mechanical analogue of the frictional sliding of a single interface from which elastic waves radiate. Due to wave reflection at the boundaries, the equivalent mass of the block $M=k\left(h / c_{s}\right)^{2} / 12$ is not independent of the equivalent spring stiffness $k$, where $h$ denotes the slab thickness and $c_{s}$ is the shear wave speed. Considering a non-monotonic friction law, we show that the relaxation oscillation regime is reached when the characteristic time scale of frictionless oscillations is much greater than the characteristic time of frictional memory effects: $(M / k)^{1 / 2} \gg L / V_{*}$. We combine a composite approximation of the stick-slip cycle and numerical studies to show that the interfacial relaxation oscillations result from the subtle interplay of the non-monotonic properties of the friction law driving the long stress build-up of the quasi-static phase, and the inertial control of the fast slip phase originating from the wave propagation. We discuss the geophysical consequences for earthquake mechanics, and connections between the rate-and-state and Coulomb models of friction.
\end{abstract}

Keywords: earthquake mechanics; relaxation oscillation; stick-slip; rate-and-state friction; non-monotonic; continuation; dynamical system; stress drop control.

1. Introduction

2. Formulation

2.1. Quasi-static approximation

2.2. A spring-block model

\section{Index}

3. Matched approximations of a stick-slip cycle 3.1. Monotonic law

3.2. Non-monotonic law

4. Discussion

\section{Introduction}

Since the seminal article of Brace and Byerlee [1], earthquakes are seen as recurrent stick-slip instabilities along preexisting fault planes whose frictional properties vary with the slip distance or slip rate. The basic idea relies on the transposition of the frictional stick-slip phenomenon observed on the scales of an experiment to the geophysical scales involved in earthquake mechanics and the seismic cycle. Together with this transfer of scale, frictional stick-slip in itself also constitutes an attractive and challenging kind of instability across the scales. Indeed, in addition to the obvious two time scales associated with the long period of stick which precedes the short slip phase, it is also known that memory effects influence the instantaneous response of the friction coefficient to velocity changes, and thus the stick-slip process, in relation with an intrinsic critical slip distance, say $L,[2,3]$. Other length scales, of macroscopic character, are also expected. Apart from the fault zone dimensions, we must also think of the length scales associated with the boundary conditions which drive the fault sliding.

*Corresponding author. Email: T.Putelat@damtp.cam.ac.uk

Philosophical Magazine

ISSN 1478-6435 print/ISSN 1478-6443 online @ 200x Taylor \& Francis http://www.tandf.co.uk/journals

DOI: $10.1080 / 1478643 \mathrm{YYxxxxxxxx}$ 
Over the past three decades, in addition to the slip rate and normal stress dependence, experimental rock mechanics has developed the concept of rate-and-state friction for which the memory effect of frictional sliding is modelled by a state variable whose evolution is controlled by the memory slip length $[4,5]$. From the point of view of (rock) friction mechanics, this characteristic microscopic length scale is usually interpreted as the slip required for the rejuvenation process of interfacial asperity contacts and is crucial for determining the characteristic length scale defining the critical medium stiffness below which stick-slip oscillations develop. From a geophysical perspective, combining this condition of stick-slip onset with the equivalent crack stiffness, the concept of memory length has been closely connected to that of nucleation length defined as the critical size that a slipping patch needs to attain so that its unstable spreading is triggered [6]. These two reasons have conferred great importance to the memory length and raised many concerns and debates among the geophysical community, upon which no agreement has been reached between experimental measurements, geophysical scalings and theoretical predictions.

A possible explanation of these inconsistent observations could lie with the implicit treatment of earthquakes in this reasoning as slip instabilities developing at the onset of stick-slip. An alternative view is obtained from considering the seismic cycle of a fault characterised by successive long stress build-ups followed by rapid stress drops associated with seismic radiation. This suggests that earthquakes result from a stick-slip oscillations far from criticality. In the vicinity of the stick-slip threshold, which corresponds to a Hopf bifurcation $[7,8]$, the stick-slip oscillations are sinusoidal. Further from the threshold, with its jerky temporal pattern, the seismic cycle looks like a relaxation oscillation instead.

Introduced in [9], relaxation oscillations are a particular kind of periodic orbit which occurs in singularly perturbed dynamical systems for which several well separated time scales are involved [10]. This generates fast and slow dynamics along the orbit which leads to large amplitude discontinuous oscillations. These two dynamics are usually constrained by the monotonicity changes of the so-called critical manifold which corresponds to the set of equilibria of the system defining the fast dynamics. We will see that this critical curve (or surface) coincides with the friction law.

Following [11], a frictional relaxation oscillation process has been proposed in early works [12,13] for explaining the stick-slip phenomenon of a spring-block system dragged at constant velocity. However no reference to the geometrical aspects of the relaxation oscillation dynamics was mentioned. Coulomb friction with constant static and dynamic friction coefficient was considered, a velocity dependence for the dynamic friction coefficient being introduced in [13]. It is furthermore important to realize that the Coulomb model of friction can predict neither any criterion of instability nor any dependence on the spring stiffness of the amplitude of the oscillations [14].

More recently, a relaxation oscillation mechanism has been advanced to model the discontinuous frictional fault slip due to earthquakes [15]. To the best of our knowledge, it is the first attempt at such description which relies on the concept of rate-and-state friction. The interesting additional feature put forward was to build a non-monotonic friction law from different competing micromechanisms of asperity deformation. However, no dynamics and wave radiation were taken into account in this analysis as only a quasi-static continuum model of a fault patch surrounded by an elastic body was considered.

In this paper, we demonstrate that the slipping dynamics of two slabs of equal thickness rubbing against each other, idealizing a natural fault, reduces to the dynamics of an equivalent spring-block system pulled at a constant velocity and whose inertia results from the account of elastic wave radiation from the fault and its reflection from boundaries. We are especially concerned with the effects of non-monotonicity of the rate-and-state law governing frictional sliding, monotonic laws having been the principal object of past researches. We nevertheless consider monotonic rate-and-state friction in a first stage to build matched approximations of the stick-slip cycle valid at small stiffnesses and to show the inertial control of the stick-slip oscillations. We also present a friction law for which the frictional strength saturates during stationary (or quasi-stationary) contact. In the laws most commonly employed so far, the interface strengthens indefinitely with time during stationary contact. The interfacial smoothing resulting from slip is also supposed to stop at higher slip rates which leads to a residual strength and induces a high velocity strengthening. We thus obtain a friction law whose steady-state form is 'spinodal' (i.e. $N$-shaped) and which agrees with the experimental results of Heslot et al. [16]. We thus explore the consequences of inertia and non-monotonic rate-and-state friction on the dynamics of frictional relaxation oscillations. 


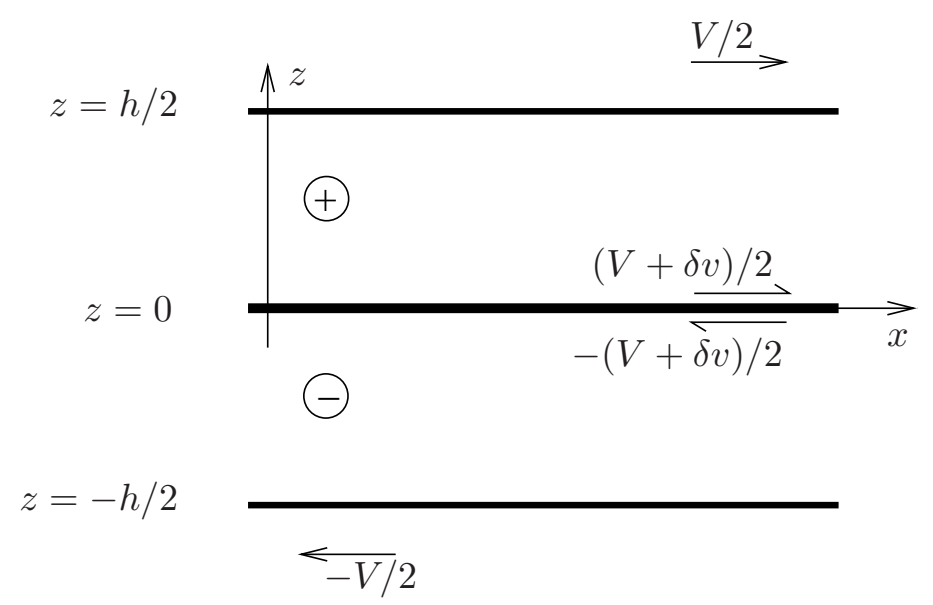

Figure 1. A single interface interface system: two elastic layers driven at constant speed $\pm V / 2$ rub against each other, elastic shear waves radiate from the frictional interface and reflect at the boundaries $z= \pm y / 2$.

Comparisons with the relaxational dynamics of a true spring-block system emphasize the particularities of the interfacial stick-slip. Geophysical consequences are discussed in view of the fact that, at leading order, the system thickness controls the stick-slip period instead of the memory length which is only involved here in the determination of the stress drop in combination with inertia.

\section{Formulation}

The problem under consideration is depicted in Figure 1. Uniform isotropic elastic layers with identical properties occupy $-h / 2<z<0$ and $0<z<h / 2$ and are denoted respectively "-" and "+". Contact between them is maintained by a uniform compressive stress $\sigma$. The upper boundary $(z=h / 2)$ is subjected to a uniform horizontal velocity $V / 2$ while the lower boundary $(z=-h / 2)$ is subjected to a uniform horizontal velocity $-V / 2$. Sliding may occur at the interface at $z=0$, according to a law of friction that is specified below. Assuming no spatial variation in the slip at the interface, the resulting displacement in either layer is horizontal and is denoted $u(z, t)$. It is antisymmetric about the interface so that only the upper layer needs to be considered explicitly.

Under the stated assumptions, the velocity field associated with $u(z, t)$ takes the form

$$
\dot{u}(z, t)=V / 2+f\left[t-(h / 2-z) / c_{s}\right]-f\left[t+(h / 2-z) / c_{s}\right],
$$

where $c_{s}$ is the shear wave speed of the medium. The rate of slip at the interface at time $t$ is then

$$
v(t)=V+2 f\left[t-h /\left(2 c_{s}\right)\right]-2 f\left[t+h /\left(2 c_{s}\right)\right] \equiv V+\delta v(t),
$$

and the rate of change of the interfacial shear stress $\tau$ is

$$
\dot{\tau}=\frac{\mathrm{G}}{c_{s}}\left\{f^{\prime}\left[t-h /\left(2 c_{s}\right)\right]+f^{\prime}\left[t+h /\left(2 c_{s}\right)\right]\right\},
$$

where $\mathrm{G}$ denotes the shear modulus.

The specification of the problem is completed with a friction law of rate-and state type. Rate-and-state friction was first introduced from rock friction experiments $[8,17-19]$. They clearly showed that the shear stress $\tau$ on a frictional interface varies with the slip rate $v$ and state variables $\phi$ which model memory effects attributed to the complex interactions of interfacial asperities controlling the relaxation response of the interface to sudden velocity changes. Note that for rocks in particular the dependence of the friction force with the normal stress $\sigma$ can be nonlinear [20]. Temperature also influences friction in various ways [5,21]. Such dependences are disregarded here, temperature and normal stress being held constant. The general 
form of the law chosen here is (e.g. [22])

$$
\left\{\begin{array}{l}
\tau=F(v, \phi ; \sigma)=\sigma \mu(v, \phi) \\
\dot{\phi}=-G(v, \phi)
\end{array}\right.
$$

with only one state variable $\phi$. Under steady-state conditions ( $v=V$ constant), $\phi$ takes the steady-state value $\phi_{s s}$ for which

$$
G\left(V, \phi_{s s}\right)=0
$$

and the associated value of the stress $\tau$ is

$$
\tau_{s s}=F\left(V, \phi_{s s}, \sigma\right) .
$$

Experimentally, it has been found in steady-state sliding that a broad variety of materials show a velocityweakening friction coefficient with a logarithmic dependence over a wide range of slip rates. Nevertheless, it has also been observed that friction behaves non-monotonically with velocity [16, 20, 23-25]. Further details and references can be found in $[4,5,21,22]$ for instance.

While certain aspects will be pursued in relation to the general law (3) in the sequel, emphasis will be placed on the Dieterich aging law

$$
\left\{\begin{array}{l}
\tau=\sigma\left[a_{*}+a \ln \left(v / V_{*}\right)+b \ln \left(V_{*} \phi / L\right)\right] \\
\dot{\phi}=1-v \phi / L
\end{array}\right.
$$

for which the state variable is interpreted as a characteristic contact lifetime of rejuvenation of the interfacial contact population. The critical slip distance $L$ is the memory length required for the interface to relax towards a new steady state in response to a velocity jump. Although this law reproduces a wide range of friction and earthquake phenomenology $[4,5]$, it suffers badly from its logarithmic singularity as $v \rightarrow 0$ in $(6)_{1}$, the absence of saturation of $\phi$ as $t \rightarrow \infty$, and its infinite monotonicity. We discussed these weaknesses in $[26]$ where we introduced the spinodal law

$$
\left\{\begin{array}{l}
\tau=\sigma a \sinh ^{-1}\left[\gamma_{*}\left(v / V_{*}\right)\left(c+\phi / \phi_{*}\right)^{b / a}\right] \\
\dot{\phi}=(1-\phi) / t_{* *}-|v| \phi / L
\end{array}\right.
$$

which regularises (6). The constant $\gamma_{*}=0.5 \exp \left(a_{*} / a\right)$ and reference state $\phi_{*}=\left(1+t_{* *} V_{*} / L\right)^{-1}$ are defined so that the reference velocity $V_{*}$ associated with the reference friction coefficient $a_{*}$ and the material constants $a$ and $b$ are the same as for (6), in order to preserve the steady-state logarithmic velocity-weakening behaviour between the two extrema of $\tau$ introduced by the additional constants $t_{* *}$ and $c$. The (small) constant $c$ confers a residual strength to the interface at the high slip rates which are associated with a vanishing interfacial state $\phi \approx 0$. This yields a local minimum of the frictional resistance attained at

$$
V_{m} \approx(b / a-1) V_{*} / c .
$$

Above this slip rate, the frictional force increases in steady-state sliding. Another velocity-strengthening regime occurs at very low velocities, that is, below

$$
V_{M} \approx a L /\left[(b-a) t_{* *}\right],
$$

where $t_{* *}$ is the characteristic time scale of aging, which competes with the dynamic weakening process involved in the state evolution law $(7)_{2}$. Note that the interfacial state variable $\phi$ is bounded when it evolves under $(7)_{2}$, in contrast to $(6)_{2}$ : under static aging the state saturates at 1 while dynamic weakening 
(a)

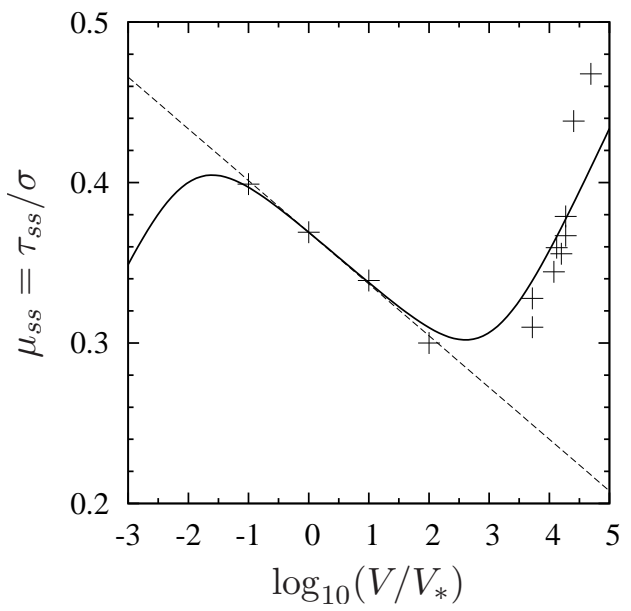

(b)

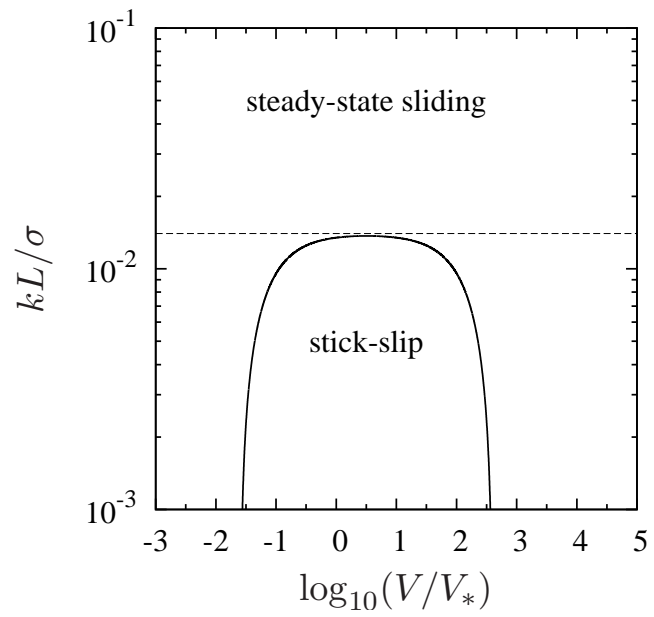

Figure 2. (a) Steady-state friction law; (b) Regime diagram: the lines correspond to the onset of stick-slip given by (10). Symbols: Dieterich law (6) (dashed line), spinodal law (7) (solid line), experimental data [16].

Table 1. Material parameter values of friction laws (6) and (7) used to fit the experimental data obtained for the frictional properties of Bristol paper board [16].

\begin{tabular}{ccccccccc}
\hline$a_{*}$ & $a$ & $b$ & $b / a$ & $b-a$ & $L$ & $V_{*}$ & $c$ & $R=t_{* *} V_{*} / L$ \\
\hline 0.369 & 0.0349 & 0.0489 & 1.4011 & 0.014 & $0.9 \times 10^{-6}$ & $10^{-6}$ & $10^{-3}$ & 100 \\
\hline
\end{tabular}

smoothes the interface towards 0. Figure 2(a) represents the steady-state forms of the laws (6) and (7) for the parameter values of table 1 and compares them to the experimental measurements of [16] obtained for Bristol paper board.

\subsection{Quasi-static approximation}

A quasi-static approximation is appropriate if the velocity of slip changes very little during the time $h / c_{s}$ taken for a wave to travel to a boundary $(z= \pm h / 2)$ and back again. In this case, the Taylor expansion $f\left[t \pm h /\left(2 c_{s}\right)\right] \approx f(t) \pm f^{\prime}(t) h /\left(2 c_{s}\right)$ combined with (1) tells that the velocity perturbation is related to the time derivative of $f(t)$ by $\delta v(t)=v(t)-V \approx-2\left(h / c_{s}\right) f^{\prime}(t)$. As also $f^{\prime}\left[t \pm h /\left(2 c_{s}\right)\right] \approx f^{\prime}(t)$, it is found that the rate of change of the interfacial shear stress $(2)$ is proportional to the velocity perturbation $\delta v(t)$, reducing the problem to the two-dimensional dynamical system

$$
\left\{\begin{array}{l}
\dot{\tau}=k[V-v(\tau, \phi)] \\
\dot{\phi}=-g(\tau, \phi) .
\end{array}\right.
$$

Note that the natural variables of the system are $\tau$ and $\phi$, while the slip rate $\dot{\delta u}=v(\tau, \phi)$ is obtained from the inversion of $(3)_{1}$ and gives the function $g(\tau, \phi)=G[v(\tau, \phi), \phi]$. The ratio $\mathrm{G} / h$ is identified as the medium stiffness $k$ and constitutes the second control parameter of the problem, in addition to the driving velocity $V$, which determine the regimes of steady-state or oscillatory sliding of the interface.

Concerning the stability of frictional sliding, it is important to realize that the properties of monotonicity of the friction law are fundamental to consider because the slope of the steady-state friction law $\tau_{s s}^{\prime}(V)$ determines the onset of stick-slip oscillations and their frequency. The linear stability analysis of the steady states $\left(\tau_{s s}, \phi_{s s}\right)$, solutions of (9) or (3) equivalently, performed by considering an infinitesimal perturbation which allows the linearisation of (9), shows that the steady states are stable only if the stiffness is large enough, greater than a critical stiffness given by

$$
k_{c}=-G_{\phi} \tau_{s s}^{\prime}
$$


More precisely, at $k=k_{c}$, a Hopf bifurcation occurs and the sliding can become oscillatory with frequency

$$
\omega_{c}^{2}=-G_{\phi}^{2} \tau_{s s}^{\prime} / F_{V},
$$

when $k \leq k_{c}$. The derivatives of $F$ and $G$ with respect to $V$ and $\phi$ are evaluated at the steady state. Expressions (10) and (11), to be defined, imply that stick-slip oscillations only arise for a velocity-weakening steady-state friction law, that is $\tau_{s s}^{\prime}(V)<0$. We thus understand the importance of behaviour changes in friction as mentioned earlier. In particular, Figure 2(b) illustrates how the existence of local extrema, like those provided by (7), bounds the stick-slip oscillations domain in the $(k, V)$-plane in comparison with the one obtained from the monotonic law (6) for which

$$
k_{c}^{D L}=\sigma(b-a) / L \quad \text { and } \quad \omega_{c}^{D L}=\sqrt{(b-a) / a}(V / L) .
$$

For the spinodal law (7), these quantities form good approximations of the corresponding $k_{c}$ and $\omega_{c}$ in the heart of the velocity-weakening region. In this regime, $k_{c}$ scales like $\sigma / L$, in proportion to the velocityweakening logarithmic rate $b-a=\mathcal{O}\left(10^{-2}\right)$. It is therefore crucial to remember that the microstructure memory length $L$ controls the onset of stick-slip via the value of $k_{c}$, the normal pressure being given. Recalling that the criterion of instability can be read as $h>\mathrm{G} / k_{c} \equiv h_{c}$, we conclude that the occurrence of stick-slip requires a macrostructure large enough to diminish its stiffness to a value sufficiently low that the medium can react to a perturbation with a delay to a forcing at constant rate $V$. In other words, stick-slip oscillations are the result of the interplay between the macro-length $h$ which is the size of the sliding system and the micro-length $L$ charateristic of the interfacial asperity microstructure. The sliding is unstable if the ratio of these two length scales satisfies

$$
L / h<(b-a) \sigma / \mathrm{G} \text {. }
$$

In terms of earthquake mechanics, this suggests that the driving of a fault defined by the couple $(h, V)$ determines the fault activity. The length $h$ could then be seen as a characteristic length scale over which the deformation is elastic, driven by some shear at rate $V$. It is difficult for us to be more precise at this point about how to interpret and transpose to some geophysical context the criterion of sliding instability. Another interpretation is usually presented in the geophysical literature and consists in considering a slipping patch of size $L_{p}$ and building another equivalent stiffness $k_{p} \propto \mathrm{G} / L_{p}$ based on arguments derived from crack solutions of Fracture Mechanics. A nucleation length $L_{p}^{\text {nucl. }} \propto \mathrm{G} L /((b-a) \sigma)$ is then deduced (see $[5,6,21,27]$ for instance). This nucleation length is defined as the critical size from which the patch expansion is unstable. The identification of the memory and nucleation lengths $L$ and $L_{p}^{\text {nucl. }}$ for natural faults has been a central issue for earthquake source mechanics and earthquake prediction and is still a matter of debate.

Another problem concerns the earthquake recurrence time which corresponds here to the period of stickslip oscillations. Equation (11) gives the analytical estimation $2 \pi / \omega_{c}$, at the instability onset. We learn from the expression of $\omega_{c}^{\mathrm{DL}}$ that the period scales like the state relaxation time $\tau_{\phi}=L / V$. This illustrates how the memory effects control the slip event recurrence at the stick-slip threshold. But, to complete the picture, numerical calculations of the period are necessary to obtain its dependence on the stiffness $k$ for a given friction law. Nevertheless, we show later in this paper that more information can be gained from asymptotic analysis of stick-slip as the stiffness is reduced in the limit $k L / \sigma \rightarrow 0$.

A major observable characterising earthquake mechanics is the stress drop associated with a slip event. The stress drop is indeed a fundamental quantity to predict as it is related to the radiated energy of an earthquake and also constitutes a crucial constraint on earthquake source models as it can be estimated from the analysis of seismograms. In the modelling we are considering, stress drop is directly related to the amplitude of stick-slip and is defined as, assuming Coulomb-type friction laws, $\Delta \tau=\sigma \Delta \mu$, where $\Delta \mu=$ $\mu_{\max }-\mu_{\min }$ is the difference between the maximum and minimum values of the friction coefficient along one stick-slip cycle. The stick-slip amplitude can be calculated from a weakly nonlinear analysis performed in the vicinity of the Hopf bifurcation [8,28,29]. In [29], we showed that the super or subcritical nature 
of the Hopf bifurcation depends strongly on the analytical details of the definition of the friction law (3). More important, we also demonstrated that the block inertia of a spring-block system cannot be neglected in the weakly nonlinear analysis. Moreover, during one cycle, the mass indeed reaches velocities large enough for the quasi-static approximation to break down, even at very slow forcing [14]. Considering (6) first, we go further in this paper by constructing a composite approximation of the stick-slip cycle in order to complete the description of the stick-slip phases as described in [14] and give a better understanding of the role of inertia in the control of friction instabilities. To introduce inertia in the single interface problem, we need to take into account wave radiation as we show now.

\section{$2.2 \quad$ A spring-block model}

A first approximation which contains allowance for the inertia of the layers is obtained by Taylor-expanding further $f\left(t \pm h / 2 c_{s}\right)$ to third order,

$$
f\left(t \pm h / 2 c_{s}\right) \sim f(t) \pm\left(h / 2 c_{s}\right) f^{\prime}(t)+\left(h / 2 c_{s}\right)^{2} f^{\prime \prime}(t) / 2 \pm\left(h / 2 c_{s}\right)^{3} f^{\prime \prime \prime}(t) / 6+\cdots,
$$

giving

$$
v(t) \approx V-2\left(h / c_{s}\right) f^{\prime}(t)-\left(h / c_{s}\right)^{3} f^{\prime \prime \prime}(t) / 12 .
$$

The second term on the right side of (14) provides a first correction to the quasi-static approximation. It has strict validity only when it is small in comparison with the first term ${ }^{1}$. This restriction is violated when the influence of the dynamics becomes significant. In this case, the approximation (14) just provides some regularisation of the system which illustrates qualitatively the influence of the actual dynamics.

In detail, it follows from the approximation (14) that

$$
\dot{\tau}=\frac{\mathrm{G}}{h}(V-v)-\frac{1}{12} \frac{\mathrm{G} h}{c_{s}^{2}} \ddot{v} .
$$

After time integration, admitting no pre-stress $\tau(0)=0$, we obtain the equation of motion

$$
M \dot{v}=k(V t-x(t))-\tau(t),
$$

of a block of mass (to be precise, a surface density of mass)

$$
M=\mathrm{G} h /\left(12 c_{s}^{2}\right),
$$

pulled at a constant rate $V$ with a spring of stiffness per unit area $k=\mathrm{G} / h$ where $x=\int_{0}^{t} v\left(t^{\prime}\right) d t^{\prime}$ is the position of the block of velocity $v(t)$.

Using the dimensions of time $L / V_{*}$, length $L$ and stress $\sigma$, the dimensionless form of equation (16) is, with a slight abuse of notation, $m \ddot{x}=\kappa(V t-x)-\mu$ where the dimensionless mass and stiffness are

$$
m=M V_{*}^{2} /(\sigma L) \text { and } \quad \kappa=k L / \sigma .
$$

Writing now the dimensionless spring force $y=\kappa(V t-x)$, its time derivative with the dimensionless form of (16) and the state evolution law (3) $)_{2}$ constitute the dynamical system which determines the sliding of the interface

$$
\left\{\begin{aligned}
\dot{y} & =\kappa(V-v), \\
\dot{\phi} & =-G(v, \phi), \\
m \dot{v} & =y-\mu(v, \phi) .
\end{aligned}\right.
$$

\footnotetext{
${ }^{1}$ More accurately, the first term that is neglected should be small in comparison with the last term retained.
} 
The quasi-static approximation (9) is consistent with the inertialess limit $m \rightarrow 0$. Therefore the consequence of the inertia introduced by the radiation and reflection of elastic waves is to couple in a subtle way the elastic and frictional stresses. Note that this coupling is different from the radiation damping generated by seismic/acoustic waves and modelled as a linearly velocity-dependent viscous drag [30,31] or a damping force proportional to the time derivative of the acceleration [32]. With the formulation (19), there is a hierarchical relation between the variables $y, \phi$ and $v$ described as the slow, intermediate and fast variables of the system when the parameters satisfy the asymptotic limit $m \kappa \ll 1$.

Such a limit occur in geophysical or experimental contexts. In the presence of inertia, the critical stiffness of a spring-block system is $k_{c}=-G_{\phi} \tau_{s s}^{\prime}\left(1+M G_{\phi} / F_{V}\right)$, which means that $\kappa_{c}^{D L}=b-a$ represents an upper bound of $\kappa$ for velocities less than $\hat{V} \equiv \sqrt{a \sigma L / M}$, a condition met in the seismogenic part of the lithosphere. That is, we must be concerned by stiffnesses such that $\kappa=\mathcal{O}\left(10^{-2}\right)$ at most. Note however that we could build experimental conditions to explore velocities $V>\hat{V}$ so that the condition $\kappa \ll 1$ breaks down. From the definitions of the stiffness $k$ and mass $M$, we have

$$
\kappa=\frac{k L}{\sigma}=\frac{\mathrm{G}}{\sigma} \frac{L}{h} \quad \text { and } \quad m=\frac{1}{12} \frac{\mathrm{G}}{\sigma} \frac{h}{L}\left(\frac{V_{*}}{c_{s}}\right)^{2},
$$

which means that the parameters $\kappa$ and $m$ are not independent, being related by

$$
m \kappa=(\mathrm{G} / \sigma)^{2}\left(V_{*} / c_{s}\right)^{2} / 12 \equiv \epsilon .
$$

Obviously a decrease in $\kappa$ results in an increase of $m$. In tectonic settings, say a brittle crust $10 \mathrm{~km}$ thick, the lithostatic pressure is about $\sigma \sim \rho g z \sim 0.1 \mathrm{GPa}$. Typical values of constitutive rock parameters are $\mathrm{G} \sim 10 \mathrm{GPa}, \sigma \sim 0.1 \mathrm{GPa}, V_{*} \sim 10^{-6} \mathrm{~m} \mathrm{~s}^{-1}, c_{s} \sim 10^{3} \mathrm{~m} \mathrm{~s}^{-1}$, which means that $\epsilon \sim 10^{-15}$. As a result, condition $m \kappa \ll 1$ is met, the relative ordering of $m$ and $\kappa$ switching for $m=\kappa=\epsilon^{1 / 2}$. Note that a different relation between $m$ and $\kappa$ exists when we consider variations of the normal stress $\sigma$ :

$$
m \kappa^{-1}=(h / L)^{2}\left(V_{*} / c_{s}\right)^{2} / 12
$$

We disregard this case in this paper as changes in normal stress, seen as a parameter, alter the friction law in various ways [33]. In particular we expect the constant $c$ which determines the location of the local minimum of the spinodal law (7) to depend on $\sigma$ as suggested by experimental evidence [23]. Varying $\sigma$ would modify the steady-state form of (7) and affect the results quantitatively. We finally notice that decoupling $m$ and $\kappa$ in the present modelling would mean that the length scale at which the wave reflection occurs would be different from the length scale $h$ from which the system is driven. This situation is conceivable in the geophysical context ${ }^{1}$.

In the following sections we explore some consequences on the stick-slip cycle of the asymptotic limit $\kappa \ll 1$, this parameter being coupled to $m$ by (20) for the single interface problem or not for the true spring-block problem. Using the language of relaxation oscillations [10], we will see that the hierarchy between the variables $y, \phi$ and $v$ reflects the relative speeds at which the different phases of the stick-slip cycle occur.

\section{Matched approximations of a stick-slip cycle}

We first construct analytically an approximation of the stick-slip cycle for the spring-block problem subjected to the monotonic law (6), the mass being small and constant $m \ll 1$. In combination with numerical computations performed with the continuation software AUTO [34], results from the previous analysis are then used to describe the relaxation oscillation regime of the interfacial stick-slip associated with the spinodal law (7). In this section, note that dimensionless quantities are used. Numerical computations are

\footnotetext{
${ }^{1}$ It could be realised in our model if some impedance boundary condition were applied at $y= \pm h / 2$.
} 
(a)

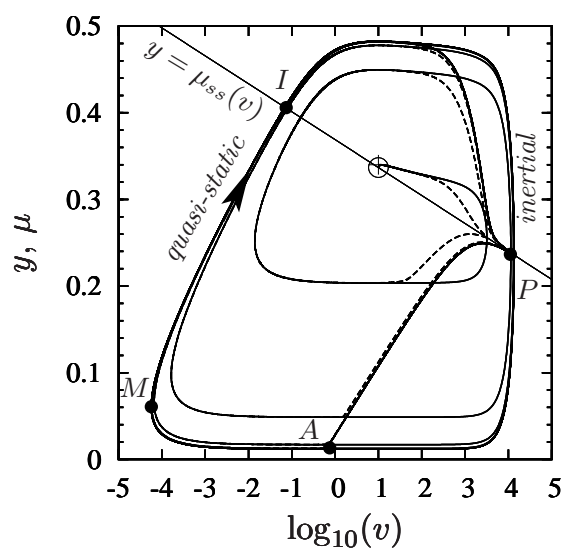

(b)

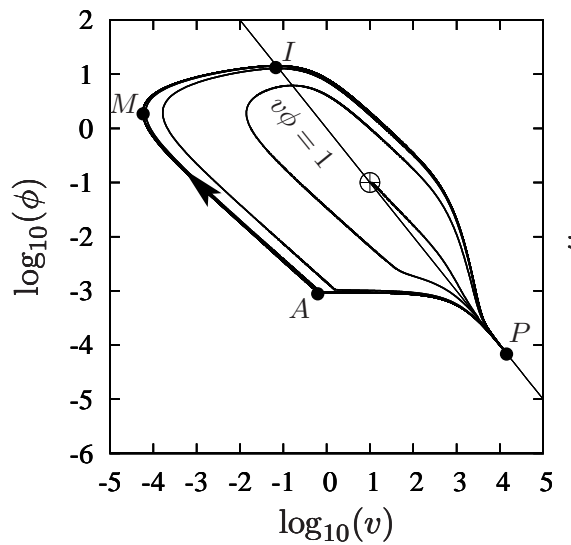

(c)

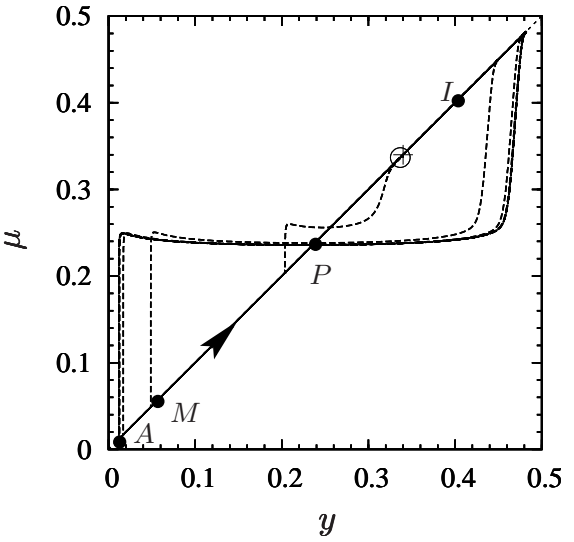

Figure 3. A stick-slip cycle trajectory for the spring-block system (19) under the Dieterich law $(6)-V=10, \kappa=2.5 \times 10^{-3}$, $m=1.1326 \times 10^{-7}$ : (a) Spring force $y$ and friction coefficient $\mu$ vs. slip rate $\log _{10}(v)$; (b) Interfacial state $\phi$ vs. slip rate $\log _{10}(v)$; (c) Friction coefficient $\mu$ vs. spring force $y$. The trajectory is computed by direct numerical time integration (MATLAB routine ode23s) from an initial condition chosen near the unstable steady state. After a transient state, a stable periodic orbit is reached. Note the effect of inertia when $y \neq \mu$. Symbols: instantaneous friction coefficient $\mu(t)$ (dashed line), spring force (thick solid line), steady-state line (thin solid line), steady-state location (o), initial condition $(+)$.

done with the material parameters of table 1. We expect the quantitative results for Bristol paper board to give a good qualitative picture of the systems behaviour when geophysical related parameter values are considered.

\subsection{Monotonic law}

We first recall the picture of spring-block stick-slip oscillations sketched by Rice and Tse [14] with the Ruina slip law defined by $G(v, \phi)=-v \phi \ln (v \phi)$ (see [4] for a friction law nomenclature and physical implications). Rice and Tse decompose a stick-slip cycle into two main phases: a long quasi-static 'stick' stage at low slip rates where the spring force builds up and is balanced by the friction force, followed by a short dynamic slip phase at high slip rates controlled by inertia and accompanied by a friction drop. During the dynamic phase, a peak in the block slip rate is attained along $\mu_{s s}(v)$ as the state quickly relaxes on $\phi_{s s}(v)$, frictional memory effects becoming instantaneous. Subsequently, an overshoot at constant state ends the cycle and determines the block 'arrest.' Figure 3 illustrates such a cycle for the Dieterich law (6). Interestingly, provided that the block acceleration is much less than $v^{2} V_{*}^{2} / L$, it is shown that the dynamic approximation leads to harmonic oscillations as the friction force slowly varies along $\mu_{s s}(v)$, eventually been considered as constant over a short range of $v$. We complete this view from building approximations of the two phases before matching them together. Note that we do not need to consider the fast intermediate stages in this process.

The quasi-static phase starts at the end of the overshoot (point $A$ ) from which friction balances the spring force $y \approx \mu(v, \phi)$ as long as $m \dot{v}=o(y)$. Hence the quasi-static slip rate follows the spring force and the interfacial state according to

$$
v=\phi^{-b / a} \exp \left[\left(y-a_{*}\right) / a\right]
$$

It is in fact more convenient to analyse this phase by considering the spring force $y$ and the block velocity $v$ as functions of the state variable $\phi$, which leads to the non-autonomous system

$$
\left\{\begin{aligned}
\mathrm{d} y / \mathrm{d} \phi & =\kappa(V-v) /(1-v \phi) \\
m \mathrm{~d} v / \mathrm{d} \phi & =[y-\mu(v, \phi)] /(1-v \phi),
\end{aligned}\right.
$$

instead of (19). Numerical time integration confirms the physical intuition which suggests that, in a first stage, the slip rate is small enough to satisfy $v \phi \ll 1$ and make the state increasing linearly in time $(\dot{\phi} \approx 1)$ until a second stage begins after crossing the steady-state line where $v \phi \gg 1$. The intersection 
point between the steady-state line and the quasi-static trajectory is denoted $I$ and satisfies $v_{I} \phi_{I}=1$ and $y_{I}=\mu_{s s}\left(v_{I}\right){ }^{1}$

In the first stage, the slip rate $v$ is also negligible compared to the driving velocity $V$. From $(22)_{1}$ this implies that the stress $y$ rises linearly with the state $\phi$. That is, by integration,

$$
y \approx \kappa V\left(\phi-\phi_{0}\right)+y_{0} .
$$

Using equation (21) we are able to find an approximate solution $y(\phi), v(\phi)$ of $(22)$, given the initial condition $y_{0}=y\left(\phi_{0}\right), v_{0}=v\left(\phi_{0}\right)$. From the point $I$, we approximate the trajectory backwards. Noticing furthermore that $y \rightarrow y_{\min }=$ cst. as $\phi \rightarrow 0$, equation (23) yields

$$
y_{\min }=\mu_{s s}\left(v_{I}\right)-\kappa V / v_{I} \text {. }
$$

Evolving backwards, at some point we will therefore encounter the minimum slip rate $V_{\text {min }}$. Indeed, during this first step, the trajectory hits a turning point $M$ in the plane $(v, y)$ which implies $\partial v / \partial y=0$. Considering $\phi$ as a function of $y$ with (23), and differentiating (21) with respect to $y$, the condition $\partial v / \partial y=0$ leads to the coordinates

$$
\phi_{M}=b /(\kappa V),
$$

which, substituted into (21) and (23), gives the coordinates $v_{M}=V_{\min }$ and $y_{M}$. The location of the point $M$ can then be evaluated once $v_{I}$ is known.

In the second quasi-static stage, the condition $v \phi \gg 1$ holds instead while $v \ll V$ still so that equation $(22)_{1}$ becomes $\mathrm{d} y / \mathrm{d} \phi \approx-\kappa V /(v \phi)$, or, by separation of variables and using $(21), \mathrm{d} \exp \left[\left(y-a_{*}\right) / a\right]=$ $-(\kappa V / b) \mathrm{d} \phi^{b / a}$. Its integration gives the trajectory $\exp \left[\left(y-a_{*}\right) / a\right]-\exp \left[\left(y_{0}-a_{*}\right) / a\right]=(\kappa V / b)\left(\phi_{0}^{b / a}-\phi^{b / a}\right)$ for any initial condition $y_{0}=y\left(\phi_{0}\right)$. Again taking the point $I$ as our initial condition, we obtain

$$
\left(y-a_{*}\right) / a=\ln \left[v_{I}^{1-b / a}+(\kappa V / b)\left(v_{I}^{-b / a}-\phi^{b / a}\right)\right],
$$

which leads to the limit $y \rightarrow y_{\max }=$ cst. as $\phi \rightarrow 0$, and so

$$
y_{\max }=\mu_{s s}\left(v_{I}\right)+a \ln \left[1+\kappa V /\left(b v_{I}\right)\right] .
$$

Already outlined in [14], the inertial phase evolves along the steady-state line as the state immediately responds to velocity changes such that friction obeys $\mu(v, \phi) \approx \mu_{s s}(v)$. During this phase, velocity changes are small enough to consider that the friction force does not vary a lot and can be approximated by its value reached at the maximum slip rate $\mu_{s s}\left(V_{\max }\right)$. As a result, system (19) reduces to $y^{\prime \prime}(\theta)+y(\theta)-\mu_{s s}\left(V_{\max }\right)=0$, having rescaled time as $\theta=t /(m / \kappa)^{1 / 2}$. If we then consider the inertial phase as a boundary value problem for which $y(0)=y_{\max }$ and $y(\pi)=y_{\min }$, the solution is

$$
y(\theta)=(\Delta y / 2) \cos \theta+\bar{y},
$$

where the stress drop is defined by $\Delta y=y_{\max }-y_{\min }$ while the mean stress reads $\bar{y}=\left(y_{\max }+y_{\min }\right) / 2$. Combined with $(19)_{1}$, equation (28) yields the inertial slip rate $v(\theta)=V+(m \kappa)^{-1 / 2}(\Delta y / 2) \sin \theta$. Note that, by construction, the inertial phase starts and ends when $v=V^{1}$ and passes through the point $P$ where the block achieves its maximum velocity $V_{\max }$. This happens half-way through the inertial phase at $\theta=\pi / 2$ which implies that the stress drop and velocity peak are related by

$$
\Delta y=2(m \kappa)^{1 / 2}\left(V_{\max }-V\right) .
$$

\footnotetext{
${ }^{1}$ Note the hypotheses $v \phi \ll 1$ or $v \phi \gg 1$ break down at the point $I$.

${ }^{1}$ This is not quite right actually. Looking at Figure (3) shows that there is a fast transition phase where $\mu(t)$ is attracted or repelled from $\mu_{s s}$ when $v>V$, the inertial phase having not started or ended yet.
} 
In addition, the mean stress coincides with the value of the friction coefficient at $V_{\max }$ :

$$
\bar{y}=\mu_{s s}\left(V_{\max }\right) .
$$

The inertial phase is thus a harmonic motion, its semi-elliptical trajectory in the plane $(v, y)$ being defined by

$$
(m \kappa)(v-V)^{2}+(y-\bar{y})^{2}=(\Delta y / 2)^{2} .
$$

Matching the quasi-static and inertial phases allows the simultaneous determination of the stress drop $\Delta y$ and mean stress $\bar{y}$ from $V_{\max }$ together with the connection point $I$ between the two quasi-static phases described above. Matching conditions directly follow from the definitions of $\Delta y$ and $y_{m}$ :

$$
y_{\max }=\bar{y}+\Delta y / 2 \quad \text { and } \quad y_{\min }=\bar{y}-\Delta y / 2 .
$$

Hence considering expressions (24), (27), (29) and (30) defines the nonlinear algebraic system we solve numerically to compute $V_{\max }$ and $v_{I}$, having being given the parameters $m, \kappa$ and $V$.

As there is no variation of kinetic energy along one entire cycle and that the work of frictional and elastic forces compensate during the quasi-static phase, the elastic energy stored in the spring during the quasi-static phase is totally dissipated by friction during the inertial stress drop. That is,

$$
\oint \mathrm{d} E_{k}=0=\int_{y_{\max }}^{y_{\min }}(\mu-y) \mathrm{d} y / \kappa \approx\left[-\mu_{s s}\left(V_{\max }\right) \Delta y+\bar{y} \Delta y\right] / \kappa .
$$

This can also be seen as a proof of equation (30).

Finally, it is important to indicate that our analysis provides the duration of the quasi-static and inertial phases. The inertial stress drop lasts a time

$$
T_{\text {slip }}=\pi(m / \kappa)^{1 / 2},
$$

while the quasi-static stress build-up takes a time

$$
T_{\text {stick }} \equiv \Delta y /(\kappa V)=2\left(V_{\max } / V-1\right)(m / \kappa)^{1 / 2} \text {. }
$$

Bifurcation diagrams presented in Figure 4 compare the analytic stick-slip cycle approximation developed here with numerical computations performed with the continuation software AUTO which allows us to follow the dynamics of system (19) under the Dieterich law (6) as the parameter $\kappa$ is varied. Overall it is found that our approximation becomes increasingly accurate at small $\kappa$ and is in error by only a few percent. However we find that the dynamics of system (19) becomes unphysical with the monotonic law (6): negative spring forces are computed as $\kappa \rightarrow 0$. This illustrates one of the major drawbacks of such monotonic friction laws. We show in the next section how considering non-monotonic friction prevents this unphysical feature.

\subsection{Non-monotonic law}

To set the scene, we are first concerned by the simpler case of relaxation oscillations in the quasi-static system (9) with the spinodal law (7). This non-monotonic regularisation of the Dieterich law (6) allows indeed the existence of periodic orbits past the Hopf birfurcation in the quasi-static approximation (9). The limit of small stiffnesses $\kappa \rightarrow 0$ renders the dynamical system (7)-(9) singular and makes the dynamics controlled by two separated time scales. In the limit $\kappa=0$, the state $\phi$ evolves on the fast time scale $t$ 
(a)

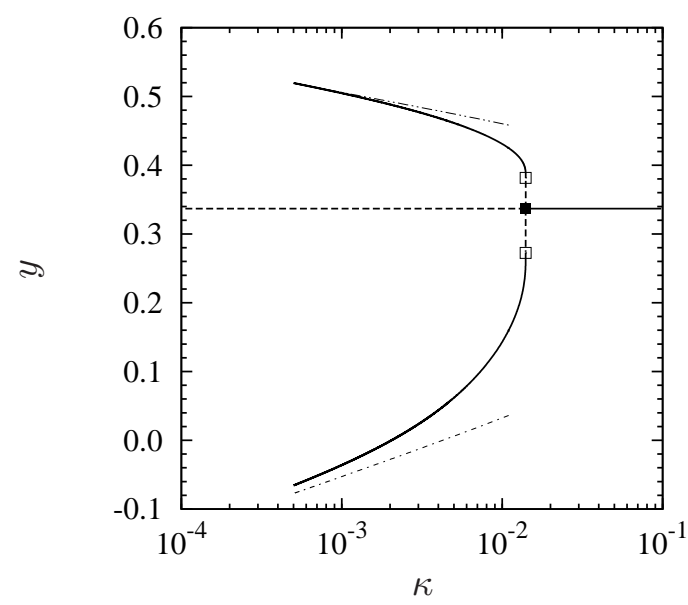

(c)

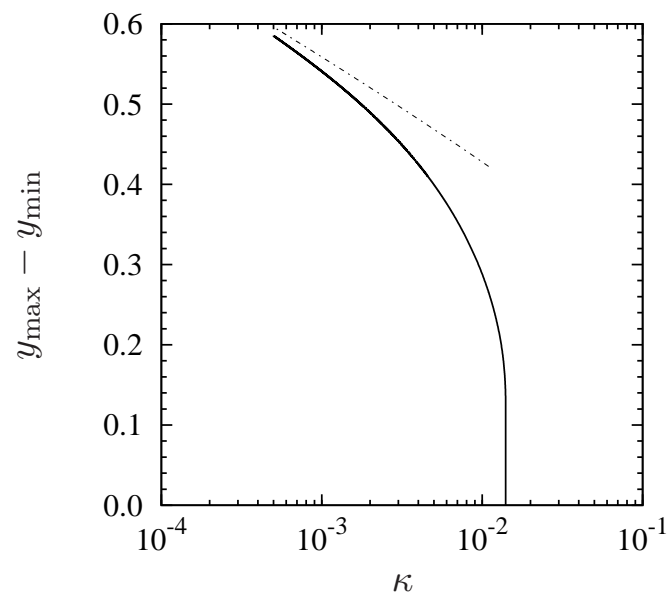

(b)

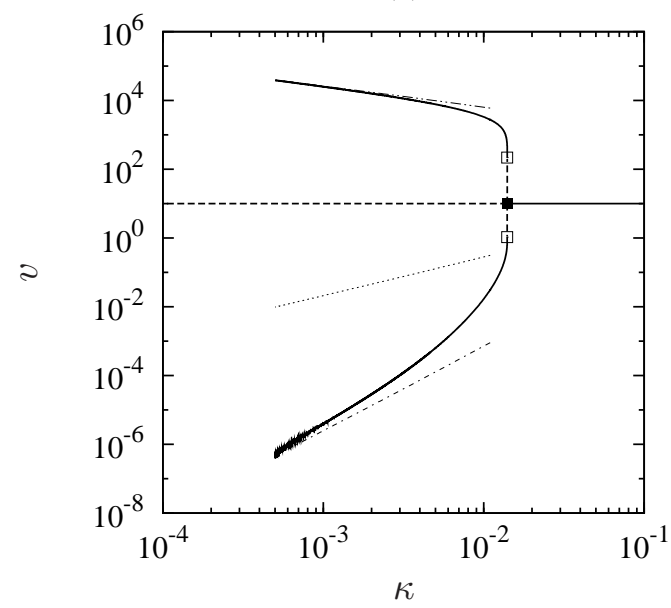

(d)

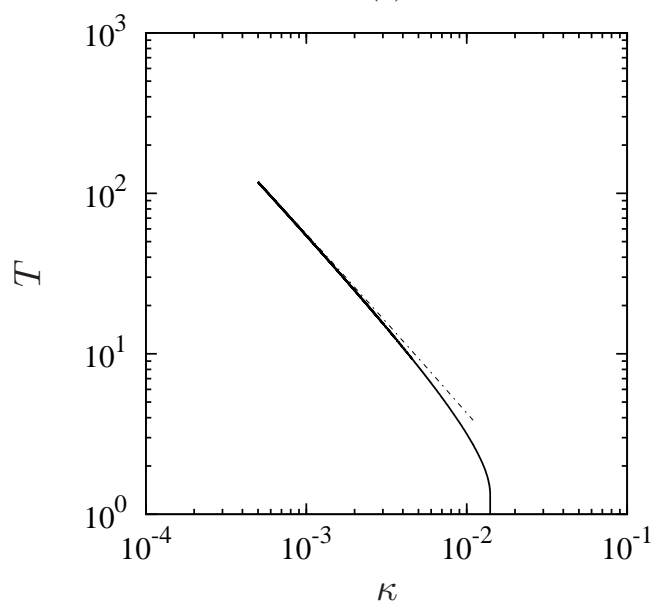

Figure 4. Bifurcation diagrams of the spring-block system (19) under the Dieterich law (6), $V=10, m=1.1326 \times 10^{-7}-$

(a) Spring force; (b) Slip rate; (c) Stress drop; (d) period. Computations performed with AUTo [34], continuation parameter $\kappa$. Symbols: unstable solution (dashed line), Hopf bifurcation (ם), saddle-node bifurcation ( $\square$ ), approximations (dashed-dotted line), slip rate $v_{I}$ (thin dotted line).

obeying the fast subsystem

$$
\left\{\begin{array}{l}
\dot{\tau}(t)=0 \\
\dot{\phi}(t)=-g(\phi ; \tau)
\end{array}\right.
$$

while the stress $\tau$ varies on the slow time scale $\hat{t}=\kappa t$ according to the slow differential-algebraic subsystem

$$
\left\{\begin{aligned}
\tau^{\prime}(\hat{t}) & =V-v(\phi, \tau) \\
0 & =g(\tau, \phi)
\end{aligned}\right.
$$

The variables $\phi$ and $\tau$ are usually referred to the fast and slow variables respectively and the equilibrium set $g(\tau, \phi)=0$ is known as the critical manifold. In the velocity-stress plane $(v, \tau)$, the critical manifold is represented by the spinodal steady-state form of (7). The qualitative behaviour of (9) can be described by combining the solutions of the subsystems (36) and (37). The asymptotic approximation when $\kappa \rightarrow 0$ of the phase trajectories of the relaxation oscillation solutions of such second-order dynamical systems is fully developed in [35].

Figure $6(\mathrm{a})$ presents in the plane $(v, \tau)$ the evolution of quasi-static periodic orbits towards the relaxation oscillation stage for smaller and smaller values of $\kappa$. As $\kappa \rightarrow 0$, we see the periodic orbit to anchor itself on the critical manifold $\tau=\mu_{s s}(v)$. From the point $P_{1}$, the first slow phase starts with the increase of 
the stress while state and slip rate are enslaved, the trajectory lying in the neighbourhood of the critical manifold. Then, the trajectory jumps at the singular point $S_{1}$ and a quasi-instantaneous rise in velocity occurs until the point $P_{2}$ is reached. A second slow phase, characterised by a stress drop, takes place down to the local minimum $S_{2}$. The quasi-static relaxation oscillation ends with a second abrupt phase over which the interface slows down before reaching $P_{1}$ again. For our purposes, to find an approximate expression for the period

$$
T_{\text {r.o. }}=(1 / \kappa) \oint \mathrm{d} \tau /(V-v),
$$

of such a relaxation oscillation, we need only calculate the duration of the phases $\left(P_{1}, S_{1}\right)$ and $\left(P_{2}, S_{2}\right)$ along the critical manifold. Considering (37), the total period is then decomposed as

$$
T_{\text {r.o. }}=T_{1}+T_{2}=(1 / \kappa) \int_{V_{1}}^{V_{M}} \frac{\tau_{s s}^{\prime}(v)}{V-v} \mathrm{~d} v+(1 / \kappa) \int_{V_{m}}^{V_{2}} \frac{\tau_{s s}^{\prime}(v)}{V-v} \mathrm{~d} v .
$$

Such decomposition was already proposed in [15]. However, since we have the law (7) we can be more explicit. During the stress build-up, which corresponds to the stick phase, $v \ll V$, we have, asymptotically

$$
T_{1} \approx(1 / \kappa) \int_{\mu_{s s}\left(V_{m}\right)}^{\mu_{s s}\left(V_{M}\right)} \mathrm{d} \tau / V=\left(y_{\max }-y_{\min }\right) /(\kappa V) .
$$

But this time, the stress drop is governed by the extrema of the spinodal law (7) which determines a constant stress drop as $\kappa \rightarrow 0$. A little algebra gives

$$
y_{\text {min }}=\mu_{s s}\left(V_{m}\right) \approx a_{*}+a \ln \left[V_{m}\left(c+1 / V_{m}\right)^{b / a}\right] \quad \text { and } \quad y_{\max }=\mu_{s s}\left(V_{M}\right) \approx a_{*}+a \ln \left[V_{M}\left((1+R) /\left(1+R V_{M}\right)\right)^{b / a}\right] \text {. }
$$

We could evaluate the duration $T_{2}$ of the stress drop similarly. But as it is negligible compared to $T_{1}$, the latter one is a good approximation of the period of quasi-static relaxation oscillations. The inversion of the spinodal law (7) also provides for the maximum and minimum slip rates during one oscillation. We find, denoting $\beta$ the ratio $b / a$ and $R$ the ratio $t_{* *} /\left(L / V_{*}\right)$,

$$
V_{\max }^{q s}=v_{s s}\left(y_{\max }\right) \approx\left(\frac{1+R}{c \beta}\right)^{\beta} \frac{(\beta-1)^{\beta-1}}{R} \text { and } V_{\min }^{q s}=v_{s s}\left(y_{\min }\right) \approx V_{m}\left(\frac{c+1 / V_{m}}{1+R}\right)^{\beta}
$$

which leads to $V_{\max }^{q s} \approx 4.4 \times 10^{4}$ and $V_{\min }^{q s} \approx 2.2 \times 10^{-4}$ with values of table 1 . It is remarkable that the driving velocity $V$ does not control the quasi-static dynamics when $\kappa \rightarrow 0$, everything being determined by the constitutive feature of the spinodal friction law. It is also worth noting that the quasi-static dynamics results from the transitions of the micromechanisms involved the physics of friction as the minimum and maximum slip rates are governed by the local extrema of the friction law. For instance, the transition between the stable strengthening due to the healing quasi-stationary processes involved at very low speed and the unstable dynamics interfacial smoothing, which fastens $\mu_{s s}\left(V_{M}\right)$, determines the maximum slip rate in combination with the residual interfacial strength responsible of the high-velocity frictional strengthening.

However, we saw with a monotonic friction law that inertia controls the slip phase and cannot be neglected. We show in the following that the behaviour of a spring-block system is a bit more subtle when a non-monotonic law is considered. Taking inertia into account makes the slip rate a fast variable when $m \ll 1$, while we refer now to the interfacial state as an intermediate variable. Two cases are to be distinguished depending on whether the parameters $\kappa$ and $m$ are connected by (20) or not. If not, as $\kappa \ll 1$, the critical manifold attracting in the phase space $(y, v, \phi)$ the slow phases of the dynamics is the set of equilibrium points $\left(\mu_{s s}(v), v, 1 / v\right)$ of the fast subsystem $\dot{y}=0, \dot{\phi}=-G(v, \phi), m \dot{v}=y-\mu(v, \phi)$. In the plane $(v, y)$, the trace of the critical manifold is represented by the steady-state friction law $y=\mu_{s s}(v)$. Figure 5(b) illustrates how the periodic orbits are finally locked on the critical manifold as $\kappa \rightarrow 0$. Figure 6 
(a)

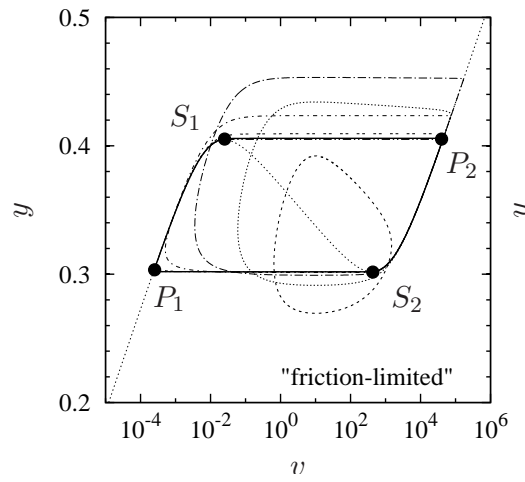

(b)

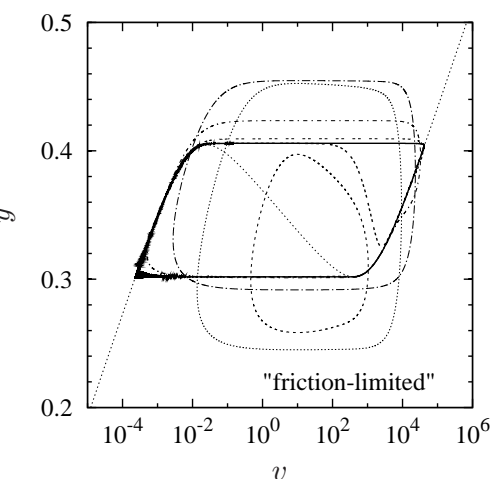

(c)

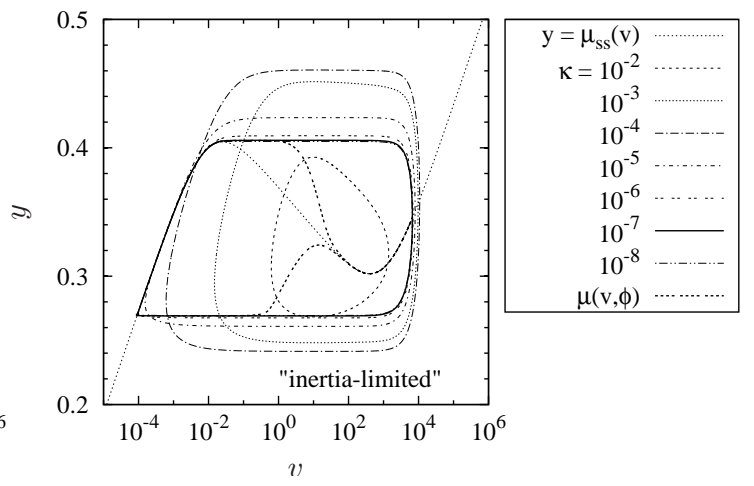

Figure 5. Towards the regimes of relaxation oscillation of the system (19) for the spinodal law (7), $V=10-$ As the stiffness $\kappa$ is reduced, the stress drop amplitude diminishes. (a) Quasi-static approximation (9); (b) Spring-block problem with constant mass $m=1.1326 \times 10^{-7} ;$ (c) Interface problem with the constraint $m \kappa=\epsilon=10^{-10}$ (20). On Figures (b) and (c) is also plotted the evolution of the instantaneous friction coefficient $\mu(v, \phi)$ during the periodic orbit for $\kappa=10^{-7}$. Note the overshoot in case (c) due to the importance of inertia. In case (b), the overshoot have already disappeared as inertial effects have become negligible. On the legend, values of $\kappa$ label the corresponding line type. Computations performed with AUTO [34], continuation parameter log $10(\kappa)$.

completes the picture by presenting bifurcation diagrams obtained with AUTO for $V=10$. It shows that the dynamics of (19) follows the quasi-static one for stiffnesses lower than $10^{-4}$. Asymptotically, although inertia is present, a regime of quasi-static relaxation oscillations is thus attained. This is understood as the combined effect of the smallness of $m$ with the limit $\kappa \rightarrow 0$ which ensures $y \approx \mu(v, \phi)$. Near the Hopf bifurcation, Figures 5 and 6 show that the amplitude of stick-slip oscillations grows rapidly with similar features as for the monotonic law (6). The Dieterich law constitutes indeed a good approximation of the frictional properties of the interface when the stick-slip amplitudes are small. In the window $10^{-4}<\kappa<\kappa_{c}$, the velocity-weakening properties of the friction law interact with its non-monotonic features to produce stick-slip oscillations whose amplitudes stay bounded, illustrating the regularizing effects of the spinodal law (7) in contrast with (6).

Considering now the constraint (20), an interesting dynamics which mixes quasi-static effects and inertial effects is found. When $m \kappa=\epsilon$ is constant, the dynamics on the slow and fast time scales $\hat{t}=\kappa t$ and $\theta=t /(m / \kappa)^{1 / 2}$ are respectively governed by

$$
\text { (a) }\left\{\begin{array} { r l } 
{ y ^ { \prime } ( \hat { t } ) } & { = V - v } \\
{ \kappa \phi ^ { \prime } ( \hat { t } ) } & { = - G ( v , \phi ) } \\
{ \epsilon v ^ { \prime } ( \hat { t } ) } & { = y - \mu ( v , \phi ) }
\end{array} \quad \text { and } \quad ( \mathrm { b } ) \left\{\begin{array}{rl}
y^{\prime}(\theta) & =\epsilon^{1 / 2}(V-v) \\
\kappa \phi^{\prime}(\theta) & =-\epsilon^{1 / 2} G(v, \phi) \\
\epsilon^{1 / 2} v^{\prime}(\theta) & =y-\mu(v, \phi) .
\end{array}\right.\right.
$$

Asymptotically, as $\kappa \rightarrow 0$, the slow dynamics is determined by (41)(a) and evolves in the vicinity of the critical manifold $y=\mu_{s s}(v)$ like for the quasi-static approximation. This is because $\epsilon \ll 1$ which makes friction balancing the elastic stress $y \approx \mu(v, \phi)$. On the contrary, the inertial phase obeys (41)(b) and behaves like the inertial phase described in section 3.1. However, as depicted in Figure 5(c), the new feature is that the maximum stress $y_{\max }$ is now established by the local maximum of the friction law $\mu_{s s}\left(V_{M}\right)$ given by (39). Matching the quasi-static and inertial phases with the condition $y_{\max }=\bar{y}+\Delta y / 2$ (cf. (32)) considering (29) and (30) which are still valid, we obtain the nonlinear relation determining the maximum slip rate

$$
\mu_{s s}\left(V_{M}\right)=\mu_{s s}\left(V_{\max }\right)+\epsilon^{1 / 2}\left(V_{\max }-V\right) .
$$

Note that this time $V_{\max }$ belongs to the high-velocity strengthening part of the spinodal law. Interestingly, the value of $V_{\max }$ is only parametrised by $\epsilon$ and thus is constant at fixed $V$. Equation (42) can be solved iteratively using (7) for $\mu_{s s}(v)$ and the dimensionless expression of (8) for $V_{M}$. For $\epsilon=10^{-10}$ and $V=10$, we find $V_{\max } \approx 6.1609 \times 10^{3}$ from (42) whereas the numerical computation by continuation of the periodic orbit gives $V_{\max } \approx 6.6131 \times 10^{3}$ for $\kappa \sim 10^{-15}$, i.e. about $7 \%$ of relative error. In the end, this inertial phase determines an overshoot controlled by the inertial harmonic stress drop $\Delta y=2 \epsilon^{1 / 2}\left(V_{\max }-V\right)$ (cf. (29)) which assigns the minimum stress $y_{\min }$. Consequently, the minimum slip rate is determined along the 
(a)

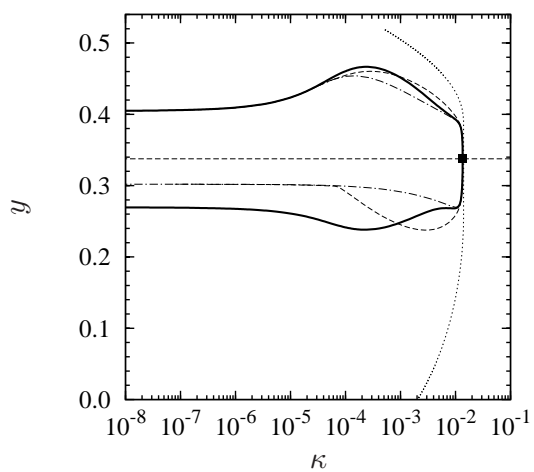

(c)

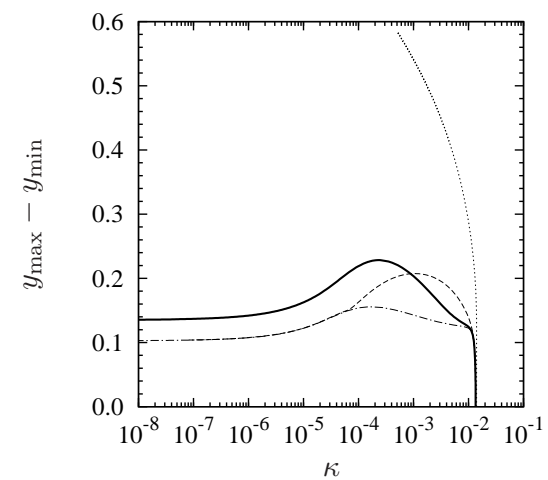

(b)

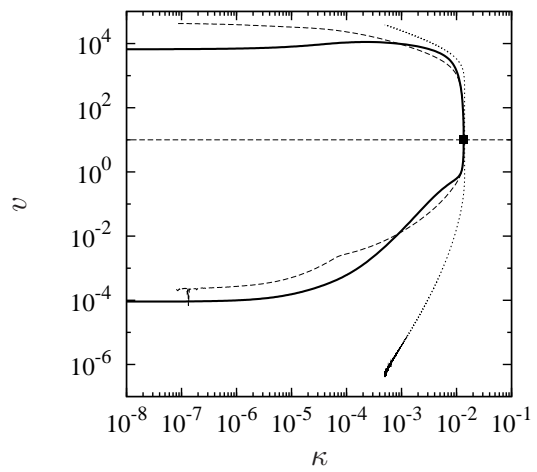

(d)

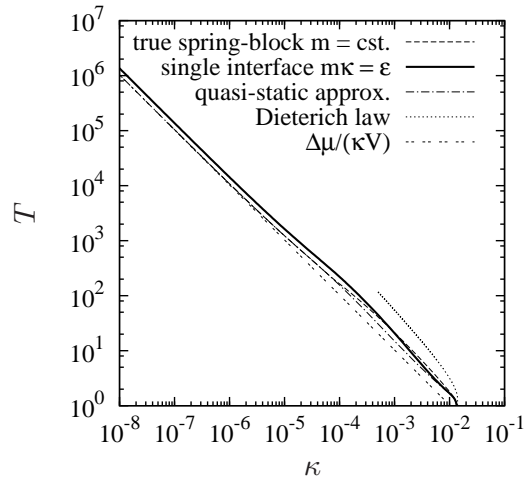

Figure 6. Bifurcation diagrams of the system (19) for the spinodal law (7), $V=10$ - Steady state and extremal amplitudes of periodic orbits are plotted for the (a) spring force; (b) slip rate; (c) stress drop; and (d) period. The nomenclature of line types is found on the legend of Figure (d). These numerical results correspond to those plotted at Figure 5.

peridic orbit as the trajectory hits at $y \approx y_{\min }$ the critical manifold of the low velocity strengthening regime of friction. From the definition of the stress drop $\Delta y$ and the maximum value of slip rate obtained above, we have

$$
\begin{aligned}
& y_{\min }=\mu_{s s}\left(V_{M}\right)-\Delta y\left(V_{\max } ; \epsilon\right), \text { and } \\
& v_{\min }=v_{s s}\left(y_{\min }\right) \approx(1+R)^{-b / a} \exp \left[\left(y_{\min }-a_{*}\right) / a\right],
\end{aligned}
$$

by combination with the approximation (A1). Numerically, these approximations give $y_{\min } \approx 0.2817$ and $v_{\text {min }} \approx 1.2730 \times 10^{-4}$, the continuation numerical method leading to $y_{\min } \approx 0.2695$ and $v_{\min } \approx 9.1074 \times 10^{-5}$; that is about $4 \%$ and $40 \%$ of relative error for $y_{\min }$ and $v_{\min }$ respectively. Although the relative error on $v_{\min }$ does not look very good, being a consequence of the rapid variation of the exponential in (44), it is worth mentioning that the approximation gives the good order of magnitude of the minimal velocity, i.e. $O\left(10^{-4}\right)$. In other words, the spinodal law predicts stick-slip cycles with minimal slip rates of order of the $\AA \mathrm{s}^{-1}$ which suggests that molecular processes should be involved in the physics of friction. Expecting more precision from our continuum model of friction would then be inappropriate, although it does agree with deformation micromechanisms based on thermo-activated diffusional creep of asperities which have been proposed by various authors to explain rate-and-state friction (see for instance $[15,22]$ ).

The dynamics of the single interface with elastic radiation and reflection modelled by (19) under spinodal friction is thus more complex than the one of a pure spring-block system for which the inertia is small and fixed. In the situation of a single frictional interface, the change of thickness of the system changes both the medium stiffness and its equivalent mass. Increasing the medium thickness reduces its stiffness while inertia rises which favors the inertial phase, the smallness of $\kappa$ emphasizing quasi-static effect during the stick phase. This latter phase then enligthens the importance of low-velocity strengthening caused by the quasi-stationary healing of the interfacial state. 


\section{Discussion}

In this article we have discussed the sliding behaviour of a single frictional interface lying between two elastic slabs of thickness $h / 2$, driven in opposite directions at constant velocity $\pm V / 2$. We have shown that the motion can be reduced to that of a block pulled at a constant velocity $V$ by a spring of stiffness $k=\mathrm{G} / h$ (the 'spring-block problem'). Previous studies assumed that the motion is quasi-static; in contrast we have shown clearly the relationship (17) between inertia in the 'spring-block problem' and acceleration of the interface arising from the generation of elastic waves and their reflection at the system boundaries.

We examined the stick-slip dynamics of the spring-block equations (19) in the small stiffness regime $\kappa=k L / \sigma \ll 1$, both for monotonic and non-monotonic rate-and-state friction laws. Two situations are considered when $\kappa \ll 1$ : the first corresponds to a true spring-block system for which the dimensionless mass $m=M V_{*}^{2} /(\sigma L)$ is constant. The second situation, $m \kappa=\epsilon,(20)$ is relevant to the frictional interface problem and arises when we consider varying slab thickness $h$ at constant normal pressure $\sigma$.

In the limit of small stiffness $\kappa \ll 1$, a hierarchy of time scales controls the system's dynamics and promotes relaxation oscillations: repeated switching between a slow quasi-static stick phase and a faster slip phase. We investigated the dynamics analytically by constructing approximate solutions in the quasistatic and fast slip phases and matching them together, and by numerical continuation through the Hopf bifurcation in which the oscillations first appear, using the bifurcation software Auto. Our leading order analysis determines, for both the true spring-block and the frictional interface problems, the stress drop $\Delta \tau=\sigma \Delta \mu$, the maximum slip rate $V_{\max }$ and the relaxation oscillation period $T$. Intriguingly, two distinct relaxation oscillation regimes can be distinguished: we referred to these as the 'friction-limited' and 'inertialimited' cases.

In the friction-limited case, inertia plays no role in the dynamics and the system evolves quasistatically even at high velocities. This case applies only when a spinodal friction law is used; the velocitystrengthening part of the spinodal law at high velocities avoids a finite time velocity blow-up. Our analysis showed that the non-monotonic interfacial properties determine the stick-slip cycle completely.

In the inertia-limited case a subtle combination of quasi-static and inertial effects governs the relaxation oscillations. Inertial effects are crucial in determining the stress drop (Eq. (29)), while an energy balance fixes the velocity peak ensuring (Eqs. (30), (33)) that the average stress equates to the friction force at the slip phase climax.

As recognised also in [14], it is remarkable that the dynamic phase is, to a very good degree of approximation, harmonic, and the block motion is resisted by an almost constant frictional force determined by the maximum block velocity. Our explanation is that in the inertial phase memory effects are almost instantaneous due to the rapid sliding, and hence the interfacial state adapts itself almost instantaneously to variations in velocity. As a result, only the steady-state friction law matters during the inertial phase, and so the interfacial state evolves under the constraint $y=\mu_{s s}(v)$.

When a spinodal friction law is used, the slow quasi-static phase follows the critical manifold of the relaxation oscillation - the low-velocity positive gradient branch of the steady-state friction law - until the local maximum of friction is reached, which defines the beginning of the slip phase. When the monotonic Dieterich friction law is used, note that the quasi-static phase is a little more complex to describe because the 'critical manifold' is reduced to a single point which divides the quasi-static evolution into two successive stages where the interface first strengthens before weakening from sliding.

The relaxation oscillation regime is fully developed when trajectories of the slow phases are confined in the neighbourhood of the 'critical manifold' which happens for the interface problem and the spring-block one when the characteristic time of the frictionless systems is much larger than the characteristic time of frictional memory effects: $(M / k)^{1 / 2} \gg L / V_{*}$. An equivalent condition $(h / L) \gg 2 \sqrt{3}\left(c_{s} / V_{*}\right)$ determines the minimum size of the system $\sqrt{3}\left(c_{s} / V_{*}\right) L$ for the interfacial relaxation oscillations to take place in relation with the frictional memory properties.

In all cases we find that the period of the stick-slip relaxation oscillation corresponds asymptotically to duration of the the quasi-static phase: $T \approx \sigma \Delta \mu /(k V)$. In the case of the spinodal law (7), $T$ can be evaluated from the friction drop $\Delta \mu=\mu_{s s}\left(V_{M}\right)-\mu_{s s}\left(V_{m}\right)$ (cf. Figure 6(d)). This approximation gives an accurate lower bound for the stick-slip period of the interfacial dynamics, even quite far from 


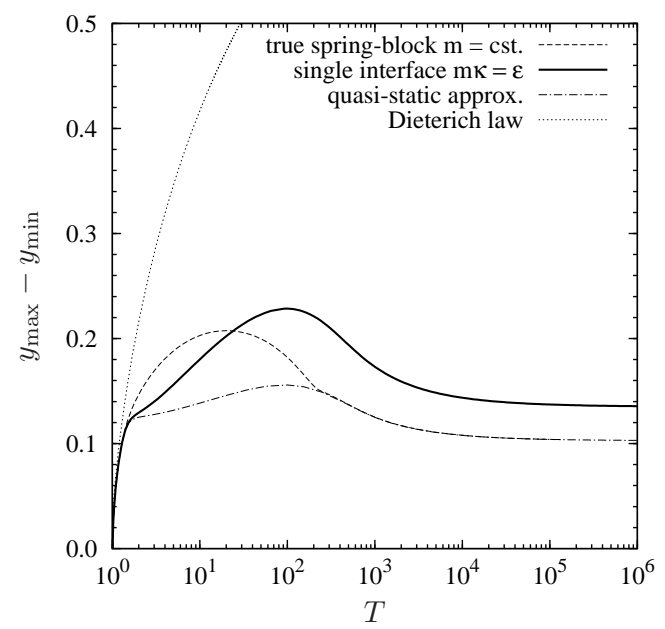

Figure 7. Stress drop vs. period of stick-slip for $V=10$ - The stress drop is independent of the stick-slip period once the relaxation oscillation regime is attained on the contrary of the unbounded growth promoted by the monotonic law.

the asymptotic relaxation oscillation regime, as shown by the numerical results in Figure 6(d). For the spinodal law, Figure 7 shows that the stress drop becomes asymptotically constant at small $\kappa$. This effect is a direct consequence of the non-monotonic character of the spinodal law (7) and differs strongly from the unbounded increase in the stress drop and oscillation period that would be predicted from a monotonic law such as (6).

We now discuss the geophysical implications of our results. Most importantly, we observe that the oscillation period is governed by the system size $h$, and not by the memory length $L$ involved at the instability onset (cf. (12)). Using geophysical orders of magnitude, we have

$$
T_{\text {r. }} \approx(\Delta \mu \sigma / \mathrm{G})(h / V) \lesssim 10^{-2} h / V \quad \text { while } \quad T_{\text {onset }} \approx 2 \pi(b / a-1)^{-1 / 2}(L / V) \lesssim 10 L / V .
$$

Thus, consideration of an earthquake as a relaxation oscillation requires no reference to a microscopic memory length, and the earthquake recurrence time should be thought of as being related to macroscopic lengthscales. A careful study of fault zones would be desirable to confront the theoretical developments of this paper with physical data, and to determine if the strain rate $V / h$ corresponds to global lithospheric strain rates or to more local strain rates associated with fault zone sizes. A simple way of doing this would be to compare observed values of $V / h$ with the ratio $\Delta \tau /\left(\mathrm{G} T_{\text {r. }}\right)$ deduced from corresponding measurements.

Due to the constraint $m \kappa=\epsilon=$ const., the interface system may generate inertia-limited relaxation oscillations with an associated stress drop

$$
\Delta \tau=2 \sigma\left[\mu_{s s}\left(V_{M}\right)-\mu_{s s}\left(V_{\max }\right)\right],
$$

which result from the interplay of the shape of the spinodal law and the inertial control of the slip phase. The stress drop emphasizes the competition between the static aging and dynamic weakening processes of the interfacial state evolution $(7)_{2}$. Through comparison with the classical stick-slip under Coulomb friction [36], our analysis links the notions of static and dynamic friction coefficients to $\mu_{s s}\left(V_{M}\right)$ and $\mu_{s s}\left(V_{\max }\right)$ respectively, and provides a physical understanding for the validity of Coulomb's model of friction. We remark (again) that only the steady-state friction coefficient is involved in determining the stress drop.

Our understanding of the inertial phase shows that the maximum slip rate becomes constant once the relaxation oscillation regime is established; it is related to the stress drop and the medium impedance by

$$
V_{\max } \approx \sqrt{3} \Delta \tau /\left(\rho c_{s}\right)+V .
$$

In the geophysical context, our analysis gives $V_{\max } \lesssim 1 \mathrm{~m} \mathrm{~s}^{-1}$. Neglecting the driving velocity $V$, it is now possible to extract the value of $\mu_{s s}\left(V_{M}\right)$ from the measurement of $\Delta \tau$ by combining (47) with (46). On 
the other hand, as the stress drop and earthquake recurrence time are related by $\Delta \tau=\mathrm{G}(V / h) T_{\text {r. }}$, the

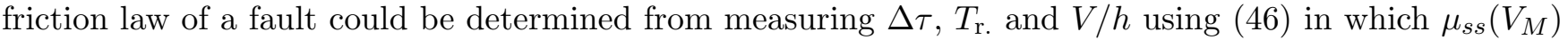
is provided by rock friction experiments.

Finally, we conclude that the spring-block equations (19) constitute a good mechanical analogue for the dynamic sliding of a frictional interface when the time scales involved are large compared to $h /\left(2 c_{s}\right)$. The quasi-static phase will satisfy this condition easily provided the system is driven slowly to ensure the "slowness condition" $V \ll(\sigma \Delta \mu / \mathrm{G}) c_{s}$. Considering geophysical values for commonly observed stress drops (1-10 bar), shear modulus (10 GPa) and wave speed $\left(10^{3} \mathrm{~m} \mathrm{~s}^{-1}\right)$ of rocks, the slowness condition is satisfied for the velocity range of Plate Tectonics. However, the analysis comes close to breaking down in the inertial phase: the condition $T_{\text {slip }} \gg h /\left(2 c_{s}\right)$ is equivalent to requiring $\pi \gg \sqrt{3}$. In conclusion we hope that our analysis motivates further experimental investigation of stick-slip dynamics, both in nature and in the laboratory.

\section{Acknowledgements}

This study has been partially supported by the European Research and Training Network DIGA (Degradation and Instabilities in Geomaterials with Application to Hazard \& Mitigation; RTN/DIGA - HPRNCT-2002-00220, Oct. 2002-Sep. 2006). TP thanks E.C. Aifantis for the funding opportunity and his interest in the subject. JHPD is supported by Newnham College, Cambridge, and by the Royal Society through a University Research Fellowship.

\section{Appendix A: Approximations of the spinodal law}

We list here useful approximations of the different steady-state sliding regimes of the spinodal law (7). At very low slip rate, the interface is rough and the state nearly constant, $\phi_{s s} \approx 1$, so that

$$
\mu_{s s}(v) \sim a_{*}+a \ln (v)+b \ln (1+R) \text { as } v \rightarrow 0 .
$$

When $v$ increases, the state is sensitive to the velocity smoothing of the interface and $\phi_{s s} \approx 1 /(1+R v)$. Thus we approximate the friction coefficient by

$$
\mu_{s s}(v) \approx a_{*}+a \ln (v)+b \ln [(1+R) /(1+R v)],
$$

which describes both the low-velocity strengthening and the logarithmic velocity-weakening. At very high slip rates, the state tends to zero, and only the residual interfacial strength $c$ remains so that friction strengthens logarithmically with the slip rate:

$$
\mu_{s s}(v) \approx a_{*}+a \ln (v)+b \ln (c) .
$$

In fact, to take into account the high-velocity transition between the velocity weakening and strengthening, we need to recognize that $\phi_{s s} / \phi_{*} \sim 1 / v$, and then

$$
\mu_{s s}(v) \approx a_{*}+a \ln (v)+b \ln (c+1 / v),
$$

because we also consider that the quasi-stationary healing processes are much longer than the process of dynamical smoothing of the interface, i.e. $R \gg 1$. 


\section{References}

[1] W.F. Brace and J.D. Byerlee, Stick slip as a mechanism for earthquakes, Science 153 (1966), pp. 990-992.

[2] J.B. Sampson et al., Friction behavior during the slip portion of the stick-slip process, J. Appl. Phys. 14 (1943), pp. 689-700.

[3] E. Rabinowicz, The intrinsic variables affecting the stick-slip process, Proc. Phys. Soc. (London) 71 (1957), pp. $668-675$.

[4] C. Marone, Laboratory-derived friction laws and their application to seismin faulting, Annu. Rev. Earth Sci. 26 (1998), pp. 643-696.

[5] C.H. Scholz, Earthquakes and friction laws, Nature 391 (1998), pp. 37-41.

[6] J.H. Dieterich, Earthquake nucleation on faults with rate-and-state dependent strength, Tectonophysics (1992), pp. 115-134.

[7] A.L. Ruina, Slip instability and state variable friction laws, J. Geophys. Res. 88 (1983), pp. 10,359-10,370.

[8] J.C. Gu et al., Slip motion and stability of a single degree of freedom elastic system with rate and state dependent friction, J. Mech. Phys. Solids 32 (1984), pp. 167-196.

[9] B. Polvan der , On relaxation oscillations, Phil. Mag. (2) (1926), pp. 978-992.

[10] V.I. Arnold et al. Bifurcation Theory and Catastrophe Theory, 2nd Springer, 1999.

[11] S. Thomas, Vibrations damped by solid friction, Phil. Mag. 9(57) (1930), pp. 329-345 7 serie.

[12] H. Blok, Fundamental mechanical aspects of boundary lubrication, S.A.E Jl. 46 (1940), pp. 54-68.

[13] B.R. Dudley and H.W. Swift, Frictional relaxation oscillations, Phil. Mag. (1949).

[14] J.R. Rice and S.T. Tse, Dynamic motion of a single degree of freedom system following a rate and state dependent friction law, J. Geophys. Res. 91(B1) (1986), pp. 521-530.

[15] Y. Estrin and Y. Bréchet, On a model of frictional sliding., Pageoph. 147 (1996), pp. 745-762.

[16] F. Heslot et al., Creep, stick-slip, and dry-friction dynamics: Experiments and a heuristic model, Phys. Rev. E 49 (1994), pp. 4973-4988.

[17] J.H. Dieterich, Modeling rock friction: 1. Experimental results and constitutive equations, J. Geophys. Res. 84 (1979), pp. $2161-68$.

[18] A.L. Ruina, Friction laws and instabilities: a quasistatic analysis of some dry frictional behavior, Brown University, 1980.

[19] J.R. Rice and A.L. Ruina, Stability of steady frictional slipping, J. App. Mech. 50 (1983), pp. 343-349.

[20] T. Shimamoto, Transition between frictional slip and ductile flow for halite shear zones at room temperature, Science 231 (1986), pp. $711-714$.

[21] C.H. Scholz The mechanics of earthquakes and faulting, 2nd Cambridge University Press, 2002.

[22] J.R. Rice, N. Lapusta, and K. Ranjith, Rate and state dependent friction and the stability of sliding between elastically deformable solids, J. Mech. Phys. Solids 49 (2001), pp. 1865-198.

[23] B.D. Kilgore, M.L. Blanpied, and J.H. Dieterich, Velocity dependent friction of granite over a wide range of conditions, Geophys. Res. Lett. 20(10) (1993), pp. 903-906.

[24] E. Rabinowicz Friction and wear of materials, 2nd John Wiley \& Sons, 1995.

[25] A. Tsutsumi and T. Shimamoto, High-velocity frictional properties of gabbro, Geophys. Res. Lett. 24 (1997), pp. 699-702.

[26] T. Putelat, J.H.P. Dawes, and J.R. Willis, Sliding interactions of two fritional interfaces, J. Mech. Phys. Solids 55(10) (2007), pp. 2073-2105.

[27] J.H. Dieterich, Implications of fault constitutive properties for earthquake prediction, Proc. Natl. Acad. Sci. USA 93 (1996), pp. 3787-3794.

[28] T. Baumberger et al., Nonlinear analysis of the stick-slip bifurcation in the creep-controlled regime of dry friction, Phys. Rev. E 51(5) (1995), pp. 4005-4010.

[29] T. Putelat, J.H.P. Dawes, and J.R. Willis, The regimes of frictional sliding of a spring-block, in preparation (200?).

[30] J.R. Rice, Spatio-temporal complexity of slip fault, J. Geophys. Res. 98 (1993), pp. 9885-9907.

[31] B.N.J. Persson Sliding Friction: Physical Principles and Applications, 2nd, NanoScience and Technology Springer, 2000.

[32] A. Johansen and D. Sornette, Acoustic radiation controls dynamic friction: Evidence from a spring-block experiment, Phys. Rev. Lett. 82 (1999), pp. 5152-5155.

[33] M. Linker and J.H. Dieterich, Effects of variable normal stress on rock friction: observations and constitutive equations, J. Geophys. Res. 97 (1992), pp. 4923-4940.

[34] E.J. Doedel, H.B. Keller, and J.P. Kernevez, Numerical analysis and control of bifurcation problems, Int. J. Bifurcation and Chaos 1(3) (1991), pp. 493-520 AUTO 2000 available via Internet from http://indy.cs.concordia.ca/auto/.

[35] E.F. Mishchenko and N.K. Rozov Differential Equations with Small Parameters and Relaxation Oscillations, Plenum Press, 1980.

[36] F.P. Bowden and D. Tabor The Friction and Lubrication of Solids, Oxford Clarendon Press, 1954. 